\title{
Two generalized $q$-exponential operators and their applications
}

Nadia Na Li* and Wei Tan

\section{*Correspondence:} lina3718@163.com

School of Mathematics and Statistics, Zhoukou Normal University, Zhoukou, 466000, P.R. China

\section{Springer}

\begin{abstract}
In this paper, we construct two generalized $q$-exponential operators with three parameters and obtain some operator identities. As applications, we give two formal extensions of the $q$-Gauss sum. An extension of the $q$-Chu-Vandermonde sums is also obtained by the operator technique. In addition, we also deduce a formal extension of the Askey-Wilson integral and a formal extension of Sears' two-term summation formula. Meanwhile, some curious q-series identities are derived.
\end{abstract}

MSC: Primary 33D15; secondary 05A15

Keywords: $q$-series; $q$-exponential operator; $q$-integral; $q$-Chu-Vandermonde sums; Askey-Wilson integral

\section{Introduction and motivation}

Following [1] we define the $q$-shifted factorial by

$$
(a ; q)_{0}=1, \quad(a ; q)_{n}=\prod_{k=0}^{n-1}\left(1-a q^{k}\right), \quad(a ; q)_{\infty}=\prod_{k=0}^{\infty}\left(1-a q^{k}\right),
$$

and $\left(a_{1}, a_{2}, \ldots, a_{m} ; q\right)_{n}=\left(a_{1} ; q\right)_{n}\left(a_{2} ; q\right)_{n} \cdots\left(a_{m} ; q\right)_{n}$, where $m$ is a positive integer and $n$ is a nonnegative integer or $\infty$. For a complex number $\alpha$, we shall also use the notation

$$
(a ; q)_{\alpha}=(a ; q)_{\infty} /\left(q^{\alpha} a ; q\right)_{\infty} .
$$

The $q$-binomial coefficients, or the Gauss coefficients, are given by

$$
\left[\begin{array}{l}
n \\
k
\end{array}\right]=\frac{(q ; q)_{n}}{(q ; q)_{k}(q ; q)_{n-k}} .
$$

The basic hypergeometric series ${ }_{r} \phi_{s}$ is defined by

$$
{ }_{r} \phi_{s}\left[\begin{array}{c}
a_{1}, a_{2}, \ldots, a_{r} \\
b_{1}, b_{2}, \ldots, b_{s}
\end{array} \mid q ; z\right]=\sum_{k=0}^{\infty} \frac{\left(a_{1}, a_{2}, \ldots, a_{r} ; q\right)_{k}}{\left(q, b_{1}, b_{2}, \ldots, b_{r} ; q\right)_{k}}\left[(-1)^{k} q^{\left(\frac{k}{2}\right)}\right]^{1+s-r} z^{k} .
$$

The usual $q$-differential operator, or $q$-derivative are defined by

$$
D_{x}\{f(x)\}=\frac{f(x)-f(q x)}{x}, \quad \theta_{x}\{f(x)\}=\frac{f\left(q^{-1} x\right)-f(x)}{q^{-1} x},
$$

(c) $2016 \mathrm{Li}$ and Tan. This article is distributed under the terms of the Creative Commons Attribution 4.0 International License (http://creativecommons.org/licenses/by/4.0/), which permits unrestricted use, distribution, and reproduction in any medium, provided you give appropriate credit to the original author(s) and the source, provide a link to the Creative Commons license, and indicate if changes were made. 
and their Leibniz rules are

$$
\begin{aligned}
& D_{x}^{n}\{f(x) g(x)\}=\sum_{k=0}^{n} q^{k(k-n)}\left[\begin{array}{l}
n \\
k
\end{array}\right] D_{x}^{k}\{f(x)\} D_{x}^{n-k}\left\{g\left(x q^{k}\right)\right\}, \\
& \theta_{x}^{n}\{f(x) g(x)\}=\sum_{k=0}^{n}\left[\begin{array}{l}
n \\
k
\end{array}\right] \theta_{x}^{k}\{f(x)\} \theta_{x}^{n-k}\left\{g\left(x q^{-k}\right)\right\},
\end{aligned}
$$

respectively. Based on this, Chen and Liu [2,3] constructed the following augmentation operator, which is of great significance for deriving identities from its special cases ( $c f$. $[4-7])$ :

$$
\begin{aligned}
& \mathbb{T}\left(b D_{x}\right)=\sum_{n=0}^{\infty} \frac{\left(b D_{x}\right)^{n}}{(q ; q)_{n}}, \\
& \mathbb{E}\left(b D_{x}\right)=\sum_{n=0}^{\infty} \frac{q^{\left(\begin{array}{c}
n \\
2
\end{array}\right)\left(b \theta_{x}\right)^{n}}}{(q ; q)_{n}} .
\end{aligned}
$$

Later, Chen and $\mathrm{Gu}[8]$ defined the Cauchy augmentation operator,

$$
\begin{aligned}
& \mathbb{T}\left(a, b D_{x}\right)=\sum_{n=0}^{\infty} \frac{(a ; q)_{n}}{(q ; q)_{n}}\left(b D_{x}\right)^{n}, \\
& \mathbb{E}\left(a,-b D_{x}\right)=\sum_{n=0}^{\infty} \frac{(a ; q)_{n}}{(q ; q)_{n}}\left(-b \theta_{x}\right)^{n} .
\end{aligned}
$$

Fang [9] and Zhang and Yang [10] considered the following finite generalized $q$-exponential operators with two parameters:

$$
\begin{aligned}
& \mathbb{T}\left[\begin{array}{c|c}
q^{-N}, w & q ; t D_{x} \\
v &
\end{array}\right]=\sum_{n=0}^{N} \frac{\left(q^{-N}, w ; q\right)_{n}}{(q, v ; q)_{n}}\left(t D_{x}\right)^{n}, \\
& \mathbb{E}\left[\begin{array}{c|c}
q^{-N}, w & q ; t \theta_{x} \\
v &
\end{array}\right]=\sum_{n=0}^{N} \frac{\left(q^{-N}, w ; q\right)_{n}}{(q, v ; q)_{n}}\left(t \theta_{x}\right)^{n} .
\end{aligned}
$$

In resent years, some authors applied the aforementioned $q$-exponential operators to give a system way of obtaining $q$-series formulas ( $c f$. [9-15]). Inspired by their work, in this paper, we construct two generalized $q$-exponential operator with three parameters and give some operator identities to further investigate the applications of the operator technique.

Our paper is organized as follows. The next section is devoted to the construction of two generalized $q$-exponential operator with three parameters and we establish some operator identities. The rest of this paper is mainly concerned with applications of these operator identities. In detail, we will give two formal extensions of the $q$-Gauss sum. An extension of the $q$-Chu-Vandermonde sums are also obtained by the operator technique. In addition, we also deduce a formal extension of the Askey-Wilson integral and a formal extension of Sears' two-term summation formula. Meanwhile, some curious $q$-series identities are derived. 


\section{Two generalized $q$-exponential operators and their operator identities}

In this section, we first of all establish the following two generalized $q$-exponential operators with three parameters:

$$
\begin{aligned}
& \mathbb{T}\left[\begin{array}{c}
u, v \\
w
\end{array} \mid q ; t D_{x}\right]=\sum_{n=0}^{\infty} \frac{(u, v ; q)_{n}}{(q, w ; q)_{n}}\left(t D_{x}\right)^{n}, \\
& \mathbb{E}\left[\begin{array}{c}
u, v \\
w
\end{array} \mid q ; t \theta_{x}\right]=\sum_{n=0}^{\infty} \frac{(u, v ; q)_{n}}{(q, w ; q)_{n}}\left(t \theta_{x}\right)^{n} .
\end{aligned}
$$

Clearly, Zhang and Yang's [10] finite $q$-exponential operators with two parameters can be considered as special cases of the operator (3) and (4) for $u=q^{-N}$ or $v=q^{-N}$. Based on the definitions for $D_{x}$ and $\theta_{x}$ and their Leibniz rules (1) and (2), we can easily verify the following explicit formulas by induction.

Lemma 1 For a nonnegative integer $k$, we have

$$
\begin{aligned}
& \theta_{x}^{k}\left\{(x t ; q)_{\infty}\right\}=(-t)^{k}(x t ; q)_{\infty}, \\
& D_{x}^{k}\left\{\frac{1}{(x t ; q)_{\infty}}\right\}=\frac{t^{k}}{(x t ; q)_{\infty}}, \\
& \theta_{x}^{k}\left\{\frac{(x v ; q)_{\infty}}{(x t ; q)_{\infty}}\right\}=t^{k} q^{-\left(\frac{k}{2}\right)}(v / t ; q)_{k} \frac{(x v ; q)_{\infty}}{\left(x t q^{-k} ; q\right)_{\infty}}, \\
& D_{x}^{k}\left\{\frac{(x v ; q)_{\infty}}{(x t ; q)_{\infty}}\right\}=t^{k}(v / t ; q)_{k} \frac{\left(x v q^{k} ; q\right)_{\infty}}{(x t ; q)_{\infty}}, \\
& \theta_{x}^{k}\left\{x^{n}\right\}= \begin{cases}(-1)^{k} x^{n-k} q^{k}\left(q^{-n} ; q\right)_{k} & \text { if } 0 \leq k \leq n, \\
0 & \text { if } k>n,\end{cases} \\
& D_{x}^{k}\left\{x^{n}\right\}= \begin{cases}x^{n-k}\left(q^{n-k+1} ; q\right)_{k} & \text { if } 0 \leq k \leq n, \\
0 & \text { if } k>n .\end{cases}
\end{aligned}
$$

Theorem 2

$$
\begin{aligned}
\mathbb{T} & \left.\begin{array}{c}
u, v \\
w
\end{array} \mid q ; t D_{x}\right]\left\{\frac{(x a ; q)_{\infty}}{(x b, x c ; q)_{\infty}}\right\} \\
& =\frac{(x a ; q)_{\infty}}{(x b, x c ; q)_{\infty}} \sum_{n, k \geq 0} \frac{(u, v ; q)_{n+k}}{(q ; q)_{n}(w ; q)_{n+k}} \frac{(a / b, x c ; q)_{k}}{(q, x a ; q)_{k}}(b t)^{k}(t c)^{n} .
\end{aligned}
$$

Proof By means of the definition (3) of $\mathbb{T}\left[\begin{array}{c}u, v \\ w\end{array} \mid q ; t D_{x}\right]$ operator and the Leibniz rule (1) of the $q$-derivative operator, we have

$$
\begin{aligned}
\mathbb{T} & {\left[\begin{array}{c}
u, v \\
w
\end{array} \mid q ; t D_{x}\right]\left\{\frac{(x a ; q)_{\infty}}{(x b, x c ; q)_{\infty}}\right\} } \\
& =\sum_{n=0}^{\infty} \frac{(u, v ; q)_{n} t^{n}}{(q, w ; q)_{n}} \sum_{k=0}^{n}\left[\begin{array}{l}
n \\
k
\end{array}\right] q^{k(k-n)} D_{x}^{k}\left\{\frac{(x a ; q)_{\infty}}{(x b ; q)_{\infty}}\right\} D_{x}^{n-k}\left\{\frac{1}{\left(q^{k} x c ; q\right)_{\infty}}\right\} .
\end{aligned}
$$


In view of Lemma 1, we can evaluate the right-hand side of the last expression in the following form:

$$
\begin{gathered}
\sum_{n=0}^{\infty} \frac{(u, v ; q)_{n} t^{n}}{(q, w ; q)_{n}} \sum_{k=0}^{n}\left[\begin{array}{l}
n \\
k
\end{array}\right] q^{k(k-n)} b^{k}(a / b ; q)_{k} \frac{\left(q^{k} x a ; q\right)_{\infty}}{(x b ; q)_{\infty}} \frac{\left(q^{k} c\right)^{n-k}}{\left(q^{k} x c ; q\right)_{\infty}} \\
\quad=\frac{(x a ; q)_{\infty}}{(x b, x c ; q)_{\infty}} \sum_{n=0}^{\infty} \frac{(u, v ; q)_{n}(c t)^{n}}{(q, w ; q)_{n}} \sum_{k=0}^{n}\left[\begin{array}{l}
n \\
k
\end{array}\right] \frac{(a / b, x c ; q)_{k}}{(x a ; q)_{k}}(b / c)^{k} .
\end{gathered}
$$

Interchanging the summation order and changing by $n \rightarrow n+k$ on the inner summation index, we get the desired result.

We remark that when $u=q^{-N}$ and $t=q / c$, the last theorem reduces to Zhang and Yang's conclusion [10]. Similar to the procedure of proving Theorem 2, we establish the following operator identity, which leads to Zhang and Yang's conclusion [10] when $u=q^{-N}$ or $v=q^{-N}$.

\section{Theorem 3}

$$
\begin{aligned}
& \mathbb{E}\left[\begin{array}{c}
u, v \\
w
\end{array} \mid q ; t \theta_{x}\right]\left\{\frac{(x a, x c ; q)_{\infty}}{(x b ; q)_{\infty}}\right\} \\
& \quad=\frac{(x a, x c ; q)_{\infty}}{(x b ; q)_{\infty}} \sum_{n, k \geq 0} \frac{(u, v ; q)_{n+k}}{(w ; q)_{n+k}(q ; q)_{n}} \frac{(a / b, q / x c ; q)_{k}}{(q, q / x b ; q)_{k}}(-1)^{n}(t c)^{n+k} q^{-\left(\frac{k}{2}\right)-n k}
\end{aligned}
$$

Letting $c=0$, the last sum with respect to $n$ in Theorem 2 and Theorem 3 vanishes because $0^{n}$ is equal to zero when $n>0$, therefore we get the following identities, respectively.

\section{Corollary 4}

$$
\begin{aligned}
& \mathbb{T}\left[\begin{array}{c}
u, v \\
w
\end{array} \mid q ; t D_{x}\right]\left\{\frac{(x a ; q)_{\infty}}{(x b ; q)_{\infty}}\right\}=\frac{(x a ; q)_{\infty}}{(x b ; q)_{\infty}}{ }_{3} \phi_{2}\left[\begin{array}{c}
u, v, a / b \\
w, x a
\end{array} \mid q ; t b\right], \\
& \mathbb{E}\left[\begin{array}{c}
u, v \\
w
\end{array} \mid q ; t \theta_{x}\right]\left\{\frac{(x a ; q)_{\infty}}{(x b ; q)_{\infty}}\right\}=\frac{(x a ; q)_{\infty}}{(x b ; q)_{\infty}}{ }_{3} \phi_{2}\left[\begin{array}{c}
u, v, a / b \\
w, q / x b
\end{array} \mid q ;-q t / x\right] .
\end{aligned}
$$

For the symmetry of $b$ and $c$ on both sides of Theorem 2, we have

$$
\sum_{n, k \geq 0} \frac{(u, v ; q)_{n+k}(a / b, x c ; q)_{k}}{(q ; q)_{n}(w ; q)_{n+k}(q, x a ; q)_{k}}(b t)^{k}(t c)^{n}=\sum_{n, k \geq 0} \frac{(u, v ; q)_{n+k}(a / c, x b ; q)_{k}}{(q ; q)_{n}(w ; q)_{n+k}(q, x a ; q)_{k}}(c t)^{k}(t b)^{n} .
$$

Similarly, by means of the symmetry of $a$ and $c$, we get from Theorem 3

$$
\begin{aligned}
& \sum_{n, k \geq 0} \frac{(u, v ; q)_{n+k}}{(w ; q)_{n+k}(q ; q)_{n}}(t c)^{n+k} \frac{(a / b, q / x c ; q)_{k}}{(q, q / x b ; q)_{k}}(-1)^{n} q^{-\left(\begin{array}{c}
k \\
2
\end{array}\right)-n k} \\
& \quad=\sum_{n, k \geq 0} \frac{(u, v ; q)_{n+k}}{(w ; q)_{n+k}(q ; q)_{n}}(t a)^{n+k} \frac{(b / c, q / x a ; q)_{k}}{(q, q / x b ; q)_{k}}(-1)^{n} q^{-\left(\begin{array}{l}
k \\
2
\end{array}\right)-n k} .
\end{aligned}
$$


Letting $n+k=m$, and extracting the coefficients of $\frac{(u, v ; q)_{m}}{(w ; q) m} t^{m}$ from both members of the last two identities, respectively, we get the following curious transformation formulas.

\section{Example 1}

$$
\begin{aligned}
& { }_{3} \phi_{1}\left[\begin{array}{c|c}
q^{-m}, a / b, x c & q ; q^{m} b / c \\
x a &
\end{array}\right]=(b / c)^{m}{ }_{3} \phi_{1}\left[\begin{array}{c}
q^{-m}, a / c, x b \\
x a
\end{array} \mid q ; q^{m} c / b\right], \\
& { }_{3} \phi_{2}\left[\begin{array}{c|c}
q^{-m}, a / b, q / x c \\
0, q / x b
\end{array} \mid q ; q\right]=(a / c)^{m}{ }_{3} \phi_{2}\left[\begin{array}{c}
q^{-m}, c / b, q / x a \\
0, q / x b
\end{array} \mid q ; q\right] \text {. }
\end{aligned}
$$

Letting $t=-w / u v c$, the sum with respect to $n$ in Theorem 3 reduces to $\frac{(w / u, w / v ; q)_{\infty}}{\left(q^{k} w, q^{-k} w / u v ; q\right)_{\infty}}$ by means of the $q$-Gauss sum

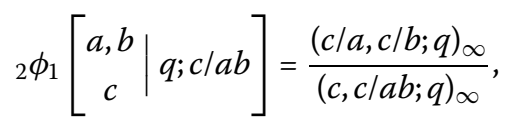

which results consequently in the following operator identity.

\section{Corollary 5}

$$
\begin{aligned}
\mathbb{E}\left[\begin{array}{c}
u, v \\
w
\end{array} \mid q ; \frac{-w}{u v c} \theta_{x}\right]\left\{\frac{(x a, x c ; q)_{\infty}}{(x b ; q)_{\infty}}\right\} \\
\quad=\frac{(x a, x c, w / u, w / b ; q)_{\infty}}{(x b, w, w / u v ; q)_{\infty}}{ }_{4} \phi_{3}\left[\begin{array}{c}
a / b, q / x c, u, v \\
0, q / x b, q u v / w
\end{array} \mid q ; q\right] .
\end{aligned}
$$

Recalling the $q$-Chu-Vandermonde sums [1], II.6,

$$
{ }_{2} \phi_{1}\left[\begin{array}{c|c}
q^{-n}, x & q ; q \\
y &
\end{array}\right]=x^{n} \frac{(y / x ; q)_{n}}{(y ; q)_{n}},
$$

and substituting $x \rightarrow x a$ into the above equation, we derive

$$
\sum_{k=0}^{n} \frac{\left(q^{-n} ; q\right)_{k} q^{k}}{(q, y ; q)_{k}} \frac{(x c ; q)_{\infty}}{\left(x a q^{k}, x b ; q\right)_{\infty}}=\frac{(x a)^{n}(y / x a ; q)_{n}(x c ; q)_{\infty}}{(y ; q)_{n}(x a, x b ; q)_{\infty}}
$$

Then applying the operator $\mathbb{T}\left[\begin{array}{c}u, v \\ w\end{array} \mid q ; t D_{x}\right]$ to both sides of (9) with respect to the variable $x$, it is trivial to see that

$$
\begin{aligned}
& \sum_{k=0}^{n} \frac{\left(q^{-n} ; q\right)_{k} q^{k}}{(q, y ; q)_{k}} \mathbb{T}\left[\begin{array}{c}
u, v \\
w
\end{array} \mid q ; t D_{x}\right]\left\{\frac{(x c ; q)_{\infty}}{\left(x a q^{k}, x b ; q\right)_{\infty}}\right\} \\
& \quad=\frac{a^{n}}{(y ; q)_{n}} \mathbb{T}\left[\begin{array}{c}
u, v \\
w
\end{array} \mid q ; t D_{x}\right]\left\{\frac{x^{n}(y / x a ; q)_{n}(x c ; q)_{\infty}}{(x a, x b ; q)_{\infty}}\right\} .
\end{aligned}
$$

Evaluating the left-hand side of the last expression in terms of Theorem 2, we show from the last equation the following identity. 
Theorem 6 For a nonnegative integer $n$, we have

$$
\begin{aligned}
& \mathbb{T}\left[\begin{array}{c}
u, v \\
w
\end{array} \mid q ; t D_{x}\right]\left\{\frac{P_{n}(x, y / a)(x c ; q)_{\infty}}{(x a, x b ; q)_{\infty}}\right\} \\
& \quad=\frac{(y ; q)_{n}}{a^{n}} \frac{(x c ; q)_{\infty}}{(x a, x b ; q)_{\infty}} \sum_{k=0}^{n} \frac{\left(q^{-n}, x a ; q\right)_{k} q^{k}}{(q, y ; q)_{k}} \sum_{i, j \geq 0} \frac{(u, v ; q)_{i+j}}{(q ; q)_{i}(w ; q)_{i+j}} \frac{\left(c / b, q^{k} x a ; q\right)_{j}}{(q, x c ; q)_{j}}(t b)^{j}\left(q^{k} t a\right)^{i},
\end{aligned}
$$

where $P_{n}(a, b)=a^{n}(b / a ; q)_{n}$.

When $y=0$, Theorem 6 reduces to the following corollary.

Corollary 7 For a nonnegative integer $n$, we have

$$
\begin{aligned}
& \mathbb{T}\left[\begin{array}{c}
u, v \\
w
\end{array} \mid q ; t D_{x}\right]\left\{\frac{x^{n}(x c ; q)_{\infty}}{(x a, x b ; q)_{\infty}}\right\} \\
& \quad=\frac{1}{a^{n}} \frac{(x c ; q)_{\infty}}{(x a, x b ; q)_{\infty}} \sum_{k=0}^{n} \frac{\left(q^{-n}, x a ; q\right)_{k}}{(q ; q)_{k}} q^{k} \sum_{i, j \geq 0} \frac{(u, v ; q)_{i+j}}{(q ; q)_{i}(w ; q)_{i+j}} \frac{\left(c / b, q^{k} x a ; q\right)_{j}}{(q, x c ; q)_{j}}(t b)^{j}\left(q^{k} t a\right)^{i} .
\end{aligned}
$$

Analogously, substituting $x \rightarrow x a$ into (7), we can reformulate it as

$$
\sum_{k=0}^{n} \frac{\left(q^{-n} ; q\right)_{k} q^{k}}{(q, y ; q)_{k}} \frac{(x b, x c ; q)_{\infty}}{\left(x a q^{k} ; q\right)_{\infty}}=\frac{(x a)^{n}(y / x a ; q)_{n}(x b, x c ; q)_{\infty}}{(y ; q)_{n}(x a ; q)_{\infty}}
$$

Applying the operator $\mathbb{E}\left[\begin{array}{c}u, v \\ w\end{array} \mid q ; t \theta_{x}\right]$ to both sides of the last expression with respect to the variable $x$, we confirm that

$$
\begin{aligned}
& \sum_{k=0}^{n} \frac{\left(q^{-n} ; q\right)_{k} q^{k}}{(q, y ; q)_{k}} \mathbb{E}\left[\begin{array}{c}
u, v \\
w
\end{array} \mid q ; t \theta_{x}\right]\left\{\frac{(x b, x c ; q)_{\infty}}{\left(x a q^{k} ; q\right)_{\infty}}\right\} \\
& \quad=\frac{a^{n}}{(y ; q)_{n}} \mathbb{E}\left[\begin{array}{c}
u, v \\
w
\end{array} \mid q ; t \theta_{x}\right]\left\{\frac{x^{n}(y / x a ; q)_{n}(x b, x c ; q)_{\infty}}{(x a ; q)_{\infty}}\right\} .
\end{aligned}
$$

Rewriting the left-hand side of the last expression by means of Theorem 3, we obtain the following identity.

Theorem 8 For a nonnegative integer $n$, we have

$$
\begin{aligned}
\mathbb{E}\left[\begin{array}{c}
u, v \\
w
\end{array} \mid q ; t \theta_{x}\right]\left\{\frac{P_{n}(x, y / a)(x b, x c ; q)_{\infty}}{(x a ; q)_{\infty}}\right\} \\
=\frac{(y ; q)_{n}}{a^{n}} \frac{(x c, x b ; q)_{\infty}}{(x a ; q)_{\infty}} \sum_{k=0}^{n} \frac{\left(q^{-n}, x a ; q\right)_{k} q^{k}}{(q, y ; q)_{k}} \\
\quad \times \sum_{i, j \geq 0} \frac{(u, v ; q)_{i+j}}{(q ; q)_{i}(w ; q)_{i+j}}(b t)^{i+j} \frac{\left(c q^{-k} / a, q / x b ; q\right)_{j}}{\left(q, q^{1-k} / x a ; q\right)_{j}}(-1)^{i} q^{-\left(\begin{array}{c}
j \\
2
\end{array}\right)-i j},
\end{aligned}
$$

where $P_{n}(a, b)=a^{n}(b / a ; q)_{n}$. 
When $b=q / x$, the inner sum with respect to $j$ vanishes, and then changing the consequent summation order, in view of the $q$-Chu-Vandermonde sum (8), we get from Theorem 8

$$
\begin{aligned}
& \mathbb{E}\left[\begin{array}{c}
u, v \\
w
\end{array} \mid q ; t \theta_{x}\right]\left\{\frac{P_{n}(x, y / a)(x c ; q)_{\infty}}{(x a ; q)_{\infty}}\right\} \\
& =\frac{x^{n}(y / x a ; q)_{n}(x c ; q)_{\infty}}{(x a ; q)_{\infty}}{ }_{2} \phi_{1}\left[\begin{array}{c}
u, v \\
w
\end{array} \mid q ;-q t / x\right] .
\end{aligned}
$$

For $t=-w x / u v q$, the right-hand side of the last identity can be evaluated by (7). We therefore establish the following operator identity.

\section{Corollary 9}

$$
\mathbb{E}\left[\begin{array}{c|c}
u, v \\
w
\end{array} \mid q ;-\frac{w x}{u v q} \theta_{x}\right]\left\{\frac{P_{n}(x, y / a)(x c ; q)_{\infty}}{(x a ; q)_{\infty}}\right\}=\frac{x^{n}(y / x a ; q)_{n}(x c, w / u, w / v ; q)_{\infty}}{(x a, w, w / u v ; q)_{\infty}} .
$$

\section{Some applications}

Throughout this section, we address the applications of the theorems and corollaries obtained in the previous section.

\subsection{Two formal generalizations of the $q$-Gauss sum}

Recalling the $q$-binomial theorem [1], II.3,

$$
\sum_{n=0}^{\infty} \frac{(a ; q)_{n}}{(q ; q)_{n}} x^{n}=\frac{(x a ; q)_{\infty}}{(x ; q)_{\infty}}
$$

multiplying $\frac{(x c ; q)_{\infty}}{(x b ; q)_{\infty}}$ to both sides of the last identity, and applying $\mathbb{T}\left[\begin{array}{c}u, v \\ { }_{w}\end{array} \mid q ; t D_{x}\right]$ to both sides of the consequent identity with respect to the parameter $x$, we have

$$
\sum_{n=0}^{\infty} \frac{(a ; q)_{n}}{(q ; q)_{n}} \mathbb{T}\left[\begin{array}{c}
u, v \\
w
\end{array} \mid q ; t D_{x}\right]\left\{\frac{x^{n}(x c ; q)_{\infty}}{(x a, x b ; q)_{\infty}}\right\}=\mathbb{T}\left[\begin{array}{c}
u, v \\
w
\end{array} \mid q ; t D_{x}\right]\left\{\frac{(x c ; q)_{\infty}}{(x, x b ; q)_{\infty}}\right\} .
$$

By means of Theorem 2 and Corollary 7, we have the following theorem.

\section{Theorem 10}

$$
\begin{aligned}
& \sum_{n=0}^{\infty} \frac{(a ; q)_{n}}{(q ; q)_{n}} a^{-n} \sum_{k=0}^{n} \frac{\left(q^{-n}, x a ; q\right)_{k}}{(q ; q)_{k}} q^{k} \sum_{i, j \geq 0} \frac{(u, v ; q)_{i+j}}{(q ; q)_{i}(w ; q)_{i+j}} \frac{\left(c / b, q^{k} x a ; q\right)_{j}}{(q, x c ; q)_{j}}(b t)^{j}\left(q^{k} t a\right)^{i} \\
& \quad=\frac{(x a ; q)_{\infty}}{(x ; q)_{\infty}} \sum_{i, j \geq 0} \frac{(u, v ; q)_{i+j}}{(q ; q)_{i}(w ; q)_{i+j}} \frac{(c, x b ; q)_{j}}{(q, x c ; q)_{j}} t^{j}(t b)^{i} .
\end{aligned}
$$

If $c=1$ and $t=w / u v b$, the sum with respect to $j$ on the right-hand side of the last theorem vanished and the sum with respect to $i$ can be evaluated by the $q$-Gauss sum (7). We find consequently the following curious summation formula where the parameter $b$ only exists in the left-hand side. 


\section{Corollary 11}

$$
\begin{aligned}
& \sum_{n=0}^{\infty} \frac{(a ; q)_{n}}{(q ; q)_{n}} a^{-n} \sum_{k=0}^{n} \frac{\left(q^{-n}, x a ; q\right)_{k}}{(q ; q)_{k}} q^{k} \\
& \quad \times \sum_{i, j \geq 0} \frac{(u, v ; q)_{i+j}}{(q ; q)_{i}(w ; q)_{i+j}} \frac{\left(1 / b, q^{k} x a ; q\right)_{j}}{(q, x ; q)_{j}}\left(\frac{w}{u v}\right)^{j}\left(\frac{q^{k} w a}{u v b}\right)^{i} \\
& =\frac{(x a, w / u, w / v ; q)_{\infty}}{(x, w, w / u v ; q)_{\infty}} .
\end{aligned}
$$

Further, if we specify with $a \mapsto 1$ in the last corollary, we find the following curious summation formula, which reduces to the $q$-Gauss sum (7) when $b=1$.

\section{Corollary 12}

$$
\sum_{i, j \geq 0} \frac{(u, v ; q)_{i+j}}{(q ; q)_{i}(w ; q)_{i+j}} \frac{(1 / b ; q)_{j}}{(q ; q)_{j}}\left(\frac{w}{u v}\right)^{j}\left(\frac{w}{u v b}\right)^{i}=\frac{(w / u, w / v ; q)_{\infty}}{(w, w / u v ; q)_{\infty}}
$$

Letting $i+j=m$, and then extracting the coefficients of $\frac{(u, v ; q) m}{(w ; q) m} t^{m}$ from two members of Theorem 10, we get the following result.

\section{Corollary 13}

$$
\begin{aligned}
\sum_{n=0}^{\infty} \frac{(a ; q)_{n}}{(q ; q)_{n}} a^{m-n} \sum_{k=0}^{n} \frac{\left(q^{-n}, x b ; q\right)_{k}}{(q ; q)_{k}} q^{(1+m) k} \\
\quad \times \sum_{j=0}^{m} \frac{\left(q^{-m}, c / b, q^{k} x a ; q\right)_{j}}{(q, x c ; q)_{j}}(-1)^{j} q^{-\left(\begin{array}{c}
j \\
2
\end{array}\right)}\left(b q^{m-k} / a\right)^{j} \\
=b^{m} \frac{(x a ; q)_{\infty}}{(x ; q)_{\infty}}{ }_{3} \phi_{1}\left[\begin{array}{c}
q^{-m}, c, x b \\
x c
\end{array} \mid q ; q^{m} / b\right]
\end{aligned}
$$

When $a=1$, the last sums with respect to $n$ and $k$ vanish because of the $q$-shift factorial $(1 ; q)_{n}$ is equal to zero when $n>0$. Then we get the following transformation formula.

\section{Example 2}

$$
{ }_{3} \phi_{1}\left[\begin{array}{c|c}
q^{-m}, c / b, x & q ; q^{m} b \\
x c &
\end{array}\right]=b^{m}{ }_{3} \phi_{1}\left[\begin{array}{c}
q^{-m}, c, x b \\
x c
\end{array} \mid q ; q^{m} / b\right] .
$$

Similarly, multiplying $\frac{(x b ; q)_{\infty}}{(x c ; q) \infty}$ to both sides of equation (10) and applying $\mathbb{E}\left[\begin{array}{c}u, v \\ w\end{array} \mid q ; t \theta_{x}\right]$ to both sides of the consequent identity with respect to the parameter $x$, we have

$$
\begin{aligned}
& \sum_{n=0}^{\infty} \frac{(a ; q)_{n}}{(q ; q)_{n}} \mathbb{E}\left[\begin{array}{c}
u, v \\
w
\end{array} \mid q ; t \theta_{x}\right]\left\{\frac{x^{n}(x, x b ; q)_{\infty}}{(x c ; q)_{\infty}}\right\} \\
& \quad=\mathbb{E}\left[\begin{array}{c}
u, v \\
w
\end{array} \mid q ; t \theta_{x}\right]\left\{\frac{(x a, x b ; q)_{\infty}}{(x c ; q)_{\infty}}\right\} .
\end{aligned}
$$


On the one hand, letting $y=0$ and substituting with $a \rightarrow c, c \rightarrow 1$ in Theorem 8 , we have

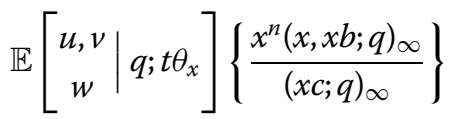

$$
\begin{aligned}
& =\frac{1}{c^{n}} \frac{(x b, x ; q)_{\infty}}{(x c ; q)_{\infty}} \sum_{k=0}^{n} \frac{\left(q^{-n}, x c ; q\right)_{k} q^{k}}{(q ; q)_{k}} \\
& \times \sum_{i, j \geq 0} \frac{(u, v ; q)_{i+j}}{(q ; q)_{i}(w ; q)_{i+j}}(b t)^{i+j} \frac{\left(q^{-k} / c, q / x b ; q\right)_{j}}{\left(q, q^{1-k} / x c ; q\right)_{j}}(-1)^{i} q^{-\left(\begin{array}{c}
j \\
2
\end{array}\right)-i j} .
\end{aligned}
$$

On the other hand, in view of Theorem 3, we get

$$
\begin{aligned}
\mathbb{E} & {\left[\begin{array}{c}
u, v \\
w
\end{array} \mid q ; t \theta_{x}\right]\left\{\frac{(x a, x b ; q)_{\infty}}{(x c ; q)_{\infty}}\right\} } \\
& =\frac{(x a, x b ; q)_{\infty}}{(x c ; q)_{\infty}} \sum_{n, k \geq 0} \frac{(u, v ; q)_{n+k}}{(w ; q)_{n+k}(q ; q)_{n}}(t b)^{n+k} \frac{(a / c, q / x b ; q)_{k}}{(q, q / x c ; q)_{k}}(-1)^{n} q^{-\left(\frac{k}{2}\right)-n k}
\end{aligned}
$$

Combining the last two expressions with (11), we get the following theorem.

\section{Theorem 14}

$$
\begin{aligned}
& \sum_{n=0}^{\infty} \frac{(a ; q)_{n}}{(q ; q)_{n}} \frac{1}{c^{n}} \sum_{k=0}^{n} \frac{\left(q^{-n}, x c ; q\right)_{k} q^{k}}{(q ; q)_{k}} \sum_{i, j \geq 0} \frac{(u, v ; q)_{i+j}(b t)^{i+j}}{(q ; q)_{i}(w ; q)_{i+j}} \frac{\left(q^{-k} / c, q / x b ; q\right)_{j}}{\left(q, q^{1-k} / x c ; q\right)_{j}}(-1)^{i} q^{-\left(\begin{array}{c}
j \\
2
\end{array}\right)-i j} \\
& \quad=\frac{(x a ; q)_{\infty}}{(x ; q)_{\infty}} \sum_{n, k \geq 0} \frac{(u, v ; q)_{n+k}}{(w ; q)_{n+k}(q ; q)_{n}}(t b)^{n+k} \frac{(a / c, q / x b ; q)_{k}}{(q, q / x c ; q)_{k}}(-1)^{n} q^{-\left(\begin{array}{c}
k \\
2
\end{array}\right)-n k} .
\end{aligned}
$$

If $a=c$, the sum with respect to $k$ on the right-hand side of the above theorem vanishes. Further setting $t=-w / u v b$ in the consequent expression, we get the following summation formula, which returns to the $q$-Gauss sum (7) when $a=1$.

\section{Corollary 15}

$$
\begin{aligned}
\sum_{n=0}^{\infty} & \frac{(a ; q)_{n}}{(q ; q)_{n}} \frac{1}{a^{n}} \sum_{k=0}^{n} \frac{\left(q^{-n}, x a ; q\right)_{k} q^{k}}{(q ; q)_{k}} \sum_{i, j \geq 0} \frac{(u, v ; q)_{i+j}}{(q ; q)_{i}(w ; q)_{i+j}} \frac{\left(q^{-k} / a, q / x b ; q\right)_{j}}{\left(q, q^{1-k} / x a ; q\right)_{j}} \\
\quad \times(-w / u v)^{i+j}(-1)^{i} q^{-\left(\begin{array}{c}
j \\
2
\end{array}\right)-i j} & \frac{(x a, w / u, w / v ; q)_{\infty}}{(x, w, w / u v ; q)_{\infty}}
\end{aligned}
$$

\subsection{A formal generalization of $q$-exponential function}

\section{Theorem 16}

$$
\sum_{n=0}^{\infty} \frac{q^{\left(\begin{array}{c}
n \\
2
\end{array}\right) x^{n}}}{(q ; q)_{n}} \sum_{k=0}^{n} \frac{\left(q^{-n}, u, v ; q\right)_{k}}{(q, w ; q)_{k}}\left(-\frac{q w}{x u v}\right)^{k}=\frac{(-x, w / u, w / v ; q)_{\infty}}{(w, w / u v ; q)_{\infty}} .
$$

Proof Recalling the $q$-exponential function [1], II.2

$$
\sum_{n=0}^{\infty} \frac{q^{\left(\begin{array}{c}
n \\
2
\end{array}\right)}}{(q ; q)_{n}} x^{n}=(-x ; q)_{\infty}
$$


and then applying $\mathbb{E}\left[\begin{array}{c}u, v \\ w\end{array} \mid q ; \frac{w}{u v} \theta_{x}\right]$ to the both sides of the last identity, we have

$$
\sum_{n=0}^{\infty} \frac{q^{\left(\begin{array}{c}
n \\
2
\end{array}\right)}}{(q ; q)_{n}} \mathbb{E}\left[\begin{array}{c}
u, v \\
w
\end{array} \mid q ; \frac{w}{u v} \theta_{x}\right]\left\{x^{n}\right\}=\mathbb{E}\left[\begin{array}{c}
u, v \\
w
\end{array} \mid q ; \frac{w}{u v} \theta_{x}\right]\left\{(-x ; q)_{\infty}\right\} .
$$

On the one hand, letting $b=0, a=-1$ and $t=w / u v$ in (6), we can write the right-hand side of (12) as

$$
\mathbb{E}\left[\begin{array}{c}
u, v \\
w
\end{array} \mid q ; \frac{w}{u v} \theta_{x}\right]\left\{(-x ; q)_{\infty}\right\}=\frac{(-x, w / u, w / v ; q)_{\infty}}{(w, w / u v ; q)_{\infty}}
$$

by means of the $q$-Gauss sum (7).

On the other hand, in view of the definition (4) and the equation of $\theta_{x}^{k}\left\{x^{n}\right\}$ obtained in Lemma 1 , we can easily verify that

$$
\mathbb{E}\left[\begin{array}{c|c}
u, v & q ; w / u v \theta_{x} \\
w
\end{array}\right]\left\{x^{n}\right\}=x^{n}{ }_{3} \phi_{2}\left[\begin{array}{c}
q^{-n}, u, v \\
w, 0
\end{array} \mid q ;-q w / u v x\right] .
$$

Inserting the last two expressions into (12), we obtain the desired result which reduces to the $q$-exponential function when $u=1$ or $v=1$.

\subsection{A generalization of $q$-Chu-Vandermonde sums}

In view of the fact that

$$
(a ; q)_{n}=\frac{(a ; q)_{\infty}}{\left(q^{n} a ; q\right)_{\infty}}
$$

and

$$
(q / a ; q)_{n}=(-a)^{-n} q^{\left(\begin{array}{c}
n+1 \\
2
\end{array}\right)} \frac{\left(q^{-n} a ; q\right)_{\infty}}{(a ; q)_{\infty}},
$$

we can reformulate the $q$-Chu-Vandermonde sums (8) as

$$
\sum_{k=0}^{n} \frac{\left(q^{-n} ; q\right)_{k} q^{k}}{(q, c ; q)_{k}} \frac{1}{\left(x q^{k} ; q\right)_{\infty}}=\frac{(-1)^{n} c^{n} q^{\left(\begin{array}{c}
n \\
2
\end{array}\right)}}{(c ; q)_{n}} \frac{\left(q^{1-n} x / c ; q\right)_{\infty}}{(x, q x / c)_{\infty}} .
$$

Applying $\mathbb{T}\left[\begin{array}{c}u, v \\ w\end{array} \mid q ; t D_{x}\right]$ to both sides of the last expression, we have

$$
\begin{aligned}
& \sum_{k=0}^{n} \frac{\left(q^{-n} ; q\right)_{k} q^{k}}{(q, c ; q)_{k}} \mathbb{T}\left[\begin{array}{c}
u, v \\
w
\end{array} \mid q ; t D_{x}\right]\left\{\frac{1}{\left(x q^{k} ; q\right)_{\infty}}\right\} \\
& \quad=\frac{(-1)^{n} c^{n} q^{\left(\frac{n}{2}\right)}}{(c ; q)_{n}} \mathbb{T}\left[\begin{array}{c}
u, v \\
w
\end{array} \mid q ; t D_{x}\right]\left\{\frac{\left(q^{1-n} x / c ; q\right)_{\infty}}{(x, q x / c)_{\infty}}\right\} .
\end{aligned}
$$

On the one hand, specifying with $a=0$ and $b=q^{k}$, we can restate (5) as

$$
\mathbb{T}\left[\begin{array}{c|c}
u, v \\
w
\end{array} \mid q ; t D_{x}\right]\left\{\frac{1}{\left(x q^{k} ; q\right)_{\infty}}\right\}=\frac{1}{\left(q^{k} x ; q\right)_{\infty}}{ }_{2} \phi_{1}\left[\begin{array}{c}
u, v \\
w
\end{array} \mid q ; q^{k} t\right] .
$$


On the other hand, in view of Theorem 2, it is not hard to derive

$$
\begin{aligned}
& \mathbb{T}\left[\begin{array}{c}
u, v \\
w
\end{array} \mid q ; t D_{x}\right]\left\{\frac{\left(q^{1-n} x / c ; q\right)_{\infty}}{(x, q x / c)_{\infty}}\right\} \\
& =\frac{\left(q^{1-n} x / c ; q\right)_{\infty}}{(x, q x / c ; q)_{\infty}} \sum_{i, k \geq 0} \frac{(u, v ; q)_{i+k}}{(q ; q)_{i}(w ; q)_{i+k}} \frac{\left(q^{1-n} / c, q x / c ; q\right)_{k}}{\left(q, q^{1-n} x / c ; q\right)_{k}} t^{i+k}(q / c)^{i}
\end{aligned}
$$

Combining the last three expression, we get the following result, which is an extension of the $q$-Chu-Vandermonde sums (8). We remark that it leads to Zhang and Yang's result ([10], Theorem 3.1), when $u$ or $v$ equals $q^{-N}$ and $t=c$.

\section{Theorem 17}

$$
\begin{aligned}
& \sum_{k=0}^{n} \frac{\left(q^{-n}, x ; q\right)_{k} q^{k}}{(q, c ; q)_{k}}{ }_{2} \phi_{1}\left[\begin{array}{c}
u, v \\
w
\end{array} \mid q ; q^{k} t\right] \\
& \quad=\frac{x^{n}(c / x ; q)_{n}}{(c ; q)_{n}} \sum_{i, k \geq 0} \frac{(u, v ; q)_{i+k}}{(q ; q)_{i}(w ; q)_{i+k}} \frac{\left(q^{1-n} / c, q x / c ; q\right)_{k}}{\left(q, q^{1-n} x / c ; q\right)_{k}} t^{i+k}(q / c)^{i} .
\end{aligned}
$$

Supposing $n=0$ and $t=w / u v$, we can reduce the previous theorem as follows.

\section{Corollary 18}

$$
\sum_{i, k \geq 0} \frac{(u, v ; q)_{i+k}}{(q ; q)_{i}(w ; q)_{i+k}} \frac{(q / c ; q)_{k}}{(q ; q)_{k}}(w / u v)^{i+k}(q / c)^{i}=\frac{(w / u, w / v ; q)_{\infty}}{(w, w / u v ; q)_{\infty}} .
$$

Letting $i+k=m$, and then extracting the coefficients of $\frac{(u, v ; q) m}{(w ; q) m} t^{m}$ from both members of Theorem 17, we get the following curious transformation formula, which results in the $q$-Chu-Vandermonde sums (8) when $m=0$.

\section{Corollary 19}

$$
{ }_{2} \phi_{1}\left[\begin{array}{c|c}
q^{-n}, x & q ; q^{m+1} \\
c &
\end{array}\right]=\frac{(c / x ; q)_{n}}{(c ; q)_{n}} x^{n}(q / c)^{m}{ }_{3} \phi_{1}\left[\begin{array}{c}
q^{-m}, q^{1-n} / c, q x / c \\
q^{1-n} x / c
\end{array} \mid q ; q^{m-1} c\right] .
$$

\subsection{A formal generalization of Sears' formula}

Recalling the Sears two-term summation formula [1], Eq. (2.10.18)

$$
\int_{c}^{d} \frac{(q t / c, q t / d, a b c d e t ; q)_{\infty}}{(a t, b t, e t ; q)_{\infty}} d_{q} t=\frac{d(1-q)(q, q d / c, c / d, a b c d, a c d e ; q)_{\infty}}{(a c, a d, b c, b d, c e, d e ; q)_{\infty}}
$$

we can restate it as

$$
\begin{aligned}
& \int_{c}^{d} \frac{(q t / c, q t / d ; q)_{\infty}}{(b t, e t ; q)_{\infty}} \frac{(a b c d e t ; q)_{\infty}}{(a t, a c d e ; q)_{\infty}} d_{q} t \\
& \quad=\frac{d(1-q)(q, q d / c, c / d ; q)_{\infty}}{(b d, c e, d e ; q)_{\infty}} \frac{(a b c d ; q)_{\infty}}{(a c, a d ; q)_{\infty}} .
\end{aligned}
$$


Further applying $\mathbb{T}\left[\begin{array}{c}u, v \\ w\end{array} \mid q ; s D_{x}\right]$ to both sides of the above identity with respect to the variable $a$, we write down immediately that

$$
\begin{gathered}
\int_{c}^{d} \frac{(q t / c, q t / d ; q)_{\infty}}{(b t, e t ; q)_{\infty}} \mathbb{T}\left[\begin{array}{c}
u, v \\
w
\end{array} \mid q ; s D_{x}\right]\left\{\frac{(a b c d e t ; q)_{\infty}}{(a t, a c d e ; q)_{\infty}}\right\} d_{q} t \\
\quad=\frac{d(1-q)(q, q d / c, c / d ; q)_{\infty}}{(b d, c e, d e ; q)_{\infty}} \mathbb{T}\left[\begin{array}{c}
u, v \\
w
\end{array} \mid q ; s D_{x}\right]\left\{\frac{(a b c d ; q)_{\infty}}{(a c, a d ; q)_{\infty}}\right\} .
\end{gathered}
$$

Applying Theorem 2 to both sides of the last identity, we therefore establish the following transformation formula.

\section{Theorem 20}

$$
\begin{gathered}
\int_{c}^{d} \frac{(q t / c, q t / d, a b c d e t ; q)_{\infty}}{(a t, b t, e t ; q)_{\infty}} \sum_{n, k=0}^{\infty} \frac{(u, v ; q)_{n+k} s^{n+k}}{(q ; q)_{n}(w ; q)_{n+k}} \frac{(a t, b t ; q)_{k}}{(q, a b c d e t ; q)_{k}}(c d e)^{k} t^{n} d_{q} t \\
\quad=\frac{d(1-q)(q, q d / c, c / d, a b c d, a c d e ; q)_{\infty}}{(a c, a d, b c, b d, c e, d e ; q)_{\infty}} \sum_{n, k=0}^{\infty} \frac{(u, v ; q)_{n+k} s^{n+k}}{(q ; q)_{n}(w ; q)_{n+k}} \frac{(b d, a d ; q)_{k}}{(q, a b c d ; q)_{k}} c^{k} d^{n} .
\end{gathered}
$$

Letting $n+k=m$, and then extracting the coefficients of $\frac{(u, v ; q) m}{(w ; q) m} s^{m}$ from both members of the last theorem, we derive the following result which leads to the Sears two-term summation formula (13) when $m=0$.

\section{Corollary 21}

$$
\begin{aligned}
& \int_{c}^{d} \frac{t^{m}(q t / c, q t / d, a b c d e t ; q)_{\infty}}{(a t, b t, e t ; q)_{\infty}}{ }_{3} \phi_{1}\left[\begin{array}{c}
q^{-m}, a t, b t \\
a b c d e t
\end{array} \mid q ; q^{m} c d e / t\right] d_{q} t \\
& \quad=\frac{d^{m+1}(1-q)(q, q d / c, c / d, a b c d, a c d e ; q)_{\infty}}{(a c, a d, b c, b d, c e, d e ; q)_{\infty}}{ }_{3} \phi_{1}\left[\begin{array}{c}
q^{-m}, a d, b d \\
a b c d
\end{array} \mid q ; q^{m} c / d\right] .
\end{aligned}
$$

\subsection{A formal generalization of Askey-Wilson integral}

Recalling the Askey-Wilson integral [13]

$$
\int_{0}^{\pi} \frac{h(\cos 2 \theta ; 1) d \theta}{h(\cos \theta ; b, c, d, x)}=\frac{2 \pi}{(q, b c, b d, c d ; q)_{\infty}} \frac{(x b c d ; q)_{\infty}}{(x b, x c, x d ; q)_{\infty}}
$$

we express it as

$$
\int_{0}^{\pi} \frac{h(\cos 2 \theta ; 1)}{h(\cos \theta ; b, c, d)} \frac{(x b ; q)_{\infty}}{\left(x e^{i \theta}, x e^{-i \theta} ; q\right)_{\infty}} d \theta=\frac{2 \pi}{(q, b c, b d, c d ; q)_{\infty}} \frac{(x b c d ; q)_{\infty}}{(x c, x d ; q)_{\infty}} .
$$

Applying $\mathbb{T}\left[\begin{array}{c}u, v \\ w\end{array} \mid q ; t D_{x}\right]$ to both sides of the above identity with respect to the variable $x$, we get

$$
\begin{gathered}
\int_{0}^{\pi} \frac{h(\cos 2 \theta ; 1)}{h(\cos \theta ; b, c, d)} \mathbb{T}\left[\begin{array}{c}
u, v \\
w
\end{array} \mid q ; t D_{x}\right]\left\{\frac{(x b ; q)_{\infty}}{\left(x e^{i \theta}, x e^{-i \theta} ; q\right)_{\infty}}\right\} d \theta \\
=\frac{2 \pi}{(q, b c, b d, c d ; q)_{\infty}} \mathbb{T}\left[\begin{array}{c}
u, v \\
w
\end{array} \mid q ; t D_{x}\right]\left\{\frac{(x b c d ; q)_{\infty}}{(x c, x d ; q)_{\infty}}\right\} .
\end{gathered}
$$


By means of Theorem 2, we get, respectively,

$$
\begin{aligned}
& \mathbb{T}\left[\begin{array}{c|c}
u, v & q ; t D_{x} \\
w & \left\{\frac{(x b ; q)_{\infty}}{\left(x e^{i \theta}, x e^{-i \theta} ; q\right)_{\infty}}\right\}
\end{array}\right. \\
& =\frac{(x b ; q)_{\infty}}{\left(x e^{i \theta}, x e^{-i \theta} ; q\right)_{\infty}} \sum_{n, k \geq 0} \frac{(u, v ; q)_{n+k}}{(q ; q)_{n}(w ; q)_{n+k}} \frac{\left(b e^{-i \theta}, x e^{-i \theta} ; q\right)_{k}}{(q, x b ; q)_{k}}\left(t e^{i \theta}\right)^{k}\left(t e^{-i \theta}\right)^{n}
\end{aligned}
$$

and

$$
\begin{aligned}
\mathbb{T} & {\left[\begin{array}{c|c}
u, v & q ; t D_{x} \\
w & \mid\left\{\frac{(x b c d ; q)_{\infty}}{(x c, x d ; q)_{\infty}}\right\}
\end{array}\right.} \\
& =\frac{(x b c d ; q)_{\infty}}{(x c, x d ; q)_{\infty}} \sum_{n, k \geq 0} \frac{(u, v ; q)_{n+k}}{(q ; q)_{n}(w ; q)_{n+k}} \frac{(b d, x d ; q)_{k}}{(q, x b c d ; q)_{k}}(t c)^{k}(t d)^{n} .
\end{aligned}
$$

Substituting the last two expressions into the identity (15), we have the following extension of the Askey-Wilson integral.

Theorem 22 For $\max \{|b|,|c|,|d|,|x|\}<1$, we have

$$
\begin{aligned}
& \int_{0}^{\pi} \frac{h(\cos 2 \theta ; 1)}{h(\cos \theta ; b, c, d, x)} \sum_{n, k \geq 0} \frac{(u, v ; q)_{n+k} t^{n+k}}{(q ; q)_{n}(w ; q)_{n+k}} \frac{\left(b e^{-i \theta}, x e^{-i \theta} ; q\right)_{k}}{(q, x b ; q)_{k}} e^{(k-n) i \theta} d \theta \\
& \quad=\frac{2 \pi(x b c d ; q)_{\infty}}{(q, b c, b d, c d, x b, x c, x d ; q)_{\infty}} \sum_{n, k \geq 0} \frac{(u, v ; q)_{n+k}}{(q ; q)_{n}(w ; q)_{n+k}} \frac{(b d, x d ; q)_{k}}{(q, x b c d ; q)_{k}}(t c)^{k}(t d)^{n} .
\end{aligned}
$$

Letting $n+k=m$, and then extracting the coefficients of $\frac{(u, v ; q)_{m}}{(w ; q)_{m}} t^{m}$ from both members of the last theorem, we thus give the following transformation formula, which leads to the Askey-Wilson integral (14) when $m=0$.

\section{Corollary 23}

$$
\begin{gathered}
\int_{0}^{\pi} \frac{h(\cos 2 \theta ; 1)}{h(\cos \theta ; b, c, d, x)} \sum_{k=0}^{m}\left[\begin{array}{c}
m \\
k
\end{array}\right] \frac{\left(b e^{-i \theta}, x e^{-i \theta} ; q\right)_{k}}{(x b ; q)_{k}} e^{(2 k-m) i \theta} d \theta \\
\quad=\frac{d^{m} 2 \pi(x b c d ; q)_{\infty}}{(q, b c, b d, c d, x b, x c, x d ; q)_{\infty}} \sum_{k=0}^{m}\left[\begin{array}{c}
m \\
k
\end{array}\right] \frac{(b d, x d ; q)_{k}}{(x b c d ; q)_{k}}(c / d)^{k} .
\end{gathered}
$$

\section{Competing interests}

The authors declare that they have no competing interests.

\section{Authors' contributions}

All authors contributed equally to the writing of this paper. All authors read and approved the final manuscript.

\section{Acknowledgements}

This research was supported by the Education Department of Henan Province (Grant No. 15A110046) and the Research Innovation Project of Zhoukou Normal University (Grant No. zknuA201410). 


\section{References}

1. Gasper, G, Rahman, M: Basic Hypergeometric Series, 2nd edn. Cambridge University Press, Cambridge (2004)

2. Chen, VYC, Liu, ZG: Parameter augmentation for basic hypergeometric series, I. J. Comb. Theory, Ser. A 80, 175-195 (1997)

3. Chen, VYC, Liu, ZG: Parameter augmentation for basic hypergeometric series, II. In: Sagan, BE, Stanley, RP (eds.) Mathematical Essays in Honor of Gian-Carlo Rota, pp. 111-129. Birkäuser, Basel (1998)

4. Liu, ZG: Some operator identities and q-series transformation formulas. Discrete Math. 265(1-3), 119-139 (2010)

5. Liu, ZG: A new proof of the Nassrallah-Rahman integral. Acta Math. Sin., Chin. Ser. 41(2), 405-410 (1998)

6. Liu, ZG: An identity of q-differential operator and its applications. J. Syst. Sci. Math. Sci. 18(3), 321-327 (1998) (in (hinese)

7. Liu, Z: An expansion formula for q-series and applications. Ramanujan J. 6(4), 429-447 (2002)

8. Chen, VYB, Gu, NSS: The Cauchy operator for basic hypergeometric series. Adv. Appl. Math. 41, 177-196 (2008)

9. Fang, JP: Some applications of q-differential operator. J. Korean Math. Soc. 47, 223-233 (2010)

10. Zhang, ZZ, Wang, JZ: Finite 9 -exponential operators with two parameters and their applications. Acta Math. Sin. 53 1007-1018 (2010) (in Chinese)

11. Fang, JP: q-Differential operator and its applications. J. Math. Anal. Appl. 332, 1393-1407 (2007)

12. Gasper, G: q-Extension of Barnes', Cauchy's and Euler's beta integrals. In: Rassias, TM (ed.) Topics in Mathematical Analysis, pp. 294-314. World Scientific, Singapore (1989)

13. Ismail, MEH, Rahman, D, Viennot, G: The combinatorics of $q$-Hermite polynomials and the Askey-Wilson integral. Eur. J. Comb. 8, 379-392 (1987)

14. Jia, Z: Two new q-exponential operator identities and their applications. J. Math. Anal. Appl. 419, 329-338 (2014)

15. Zhang, ZZ, Wang, J: Two operator identities and their applications to terminating basic hypergeometric series and q-integrals. J. Math. Anal. Appl. 312, 653-665 (2005)

\section{Submit your manuscript to a SpringerOpen ${ }^{\circ}$ journal and benefit from:}

- Convenient online submission

- Rigorous peer review

- Immediate publication on acceptance

- Open access: articles freely available online

- High visibility within the field

- Retaining the copyright to your article 